\title{
AMERICAN JOURNAL OF SCIENCE
}

\author{
[ T H I R D S E R I E S.]
} Art. I.-Measurement of the Peruvian Arc; by
E. D. Preston.*

ONE hundred and fifty years have passed since Bouguer was making his observations in the measurement of the Peruvian arc. The geodetic science of to.day is so much occupied with the slight deviations of the surface of the earth from a strictly elliptical figure, that it is hard to realize that even in the last century it was an unsettled question whether the equatorial or polar axis was the longer.

A clock, having been carried from Paris to the equator, was found to lose two minutes each day. This fact was supposed to strengthen Newton's theory that the earth was an oblate spheroid. On the other hand, Cassini's surveys in France at the beginning of the last century, indicated a prolate spheroid. It was to reconcile these two determinations that the French Academy undertook the measurements of Meridional arcs; one on a frozen river in Lapland, the other above the clonds in Per't.

Parenthetically, it should be stated, however, that the Peruvian are so-called, is not in Peru as defined by the geography

* Read before the American Association for the Advancement of Science, Toronto meeting, August, 1889. Published by permission of the Superintendent of the U. S. Coast and Geodetic Survey.

Am. Jour. Sci.-Third Series, Voi. XXXIX, No. 229.-Jan., 1890. 
of to-day, but more than a hundred miles north of it in Equador. When the results of these two expeditions were made known, the scientific world accepted Newton's theory, and all later measures have only served to confir'm it.

Let us pause just for an instant to examine the triangulation by Cassini, and the time determination by Richer. Looking at the data with our present knowledge of the accuracy attainable in the two kinds of measurement, it seems strange that the former could for a moment have cast doubt on the latter. In the first place, Cassini's results do not agree among themselves. He gives the following statement of the length of one degree in toises:

\begin{tabular}{|c|c|}
\hline$\phi$ & $\tau$ \\
\hline $49^{\circ} 5$ & 56970 \\
\hline $49 \quad 22$ & 57060 \\
\hline $47 \quad 57$ & 57098 \\
\hline
\end{tabular}

Even were there no other reason for distrusting the observations, their disagreement would almost condemm them. $\mathrm{We}$ now know that the length of one degree in latitude $49^{\circ}$ changes about ten toises per degree, so that the change of more than one hundred and thirty toises in a space of as many miles, indicates either some large error or a value for the earth's radii entirely incompatible with even the rudest observations. Besides, if the first difference were accepted, it would require the place of observation to be in a latitude very different from that known to have been the case. Therefore the triangulation in itself is not very trustworthy. Moreover any assumption in regard to the ellipticity of the meridian derived from measures not extending beyond two degrees, is extremely hazardous.

On the other hand, when we consider that Richer's clock lost two minutes daily, and that it must have been a comparatively easy matter, even at that time, to get differential time within a second, it is plain that the only somrce of error worth examining is that due to the change of the length of the pendulum. Barring accidents, and leaving to one side the effect of temperature, which must have been well understood and taken account of by the observer, the length could not have changed by nearly so much as its one-thousandth part, and as the time varies as the square root of the length of the pendulum, the time of one oscillation could not have been in error more than one-half this amount. Hence no error can be admitted that would materially change the result, and the pendulum work might have been accepted as demonstrating the oblateness of the figure. But the Academy resolved upon an independent determination, and the two expeditions were equipped. 
Bouguer set out on May 6th, 1735, and after a journey of more than a year, arrived at his destination. The party was absent about nine years, but the triangulation and base measurements were executed between December, 1736 and August, 1739. Astronomical observations to determine the amplitude of the are were made between July, 1741 and January, 1743, and the party arrived at the mouth of the Magdalena river on September 30 th of the same year. The pendulum was swung at Porto Bello on the ontward trip, and at Petit Goave, Hayti, on the return voyage. The results are incorporated in the account of the equatorial work. Three gravity determinations were made in Peru ; at the sea-level, at Quito (9,374 feet elevation), and on the summit of Pichincha (15,564 feet). Magnetic observations were also carried on, and a general study made of the natural history and physical features of the country.

We must not lose sight of the fact that the work was undertaken to decide between the relative lengths of the earth's axes. Several methods of arriving at this result were therefore considered. It was once thought to supply sufficient data to decide the whole question by the equatorial observations alone: measuring for this purpose a degree of latitude and one of longitude in the same locality. But recognizing the fact that with the means at hand, the former would be subject to an error of $1 / 1500$ th part while the latter would be uncertain by about six times as much, the preference was given to the degree of the meridian. Measures had already been made in France, and from the nature of the involute curve, formed by the intersection of the earth's radii for any given meridian, it was admitted that combining the equatorial measures with those of a middle latitude, the error to be expected in the ratio of the two axes was only 1/1440th part; and that a combination with arctic measures would reduce the error to about twothirds as much (1/2030). The errors attributed to accidental causes rest on the assumption that in each astronomical observation the observer is liable to be mistaken by three or four seconds of arc; and that in noting signals for longitude one second of time would be the error expected.

After the arc had been measured it became a matter of some difficulty to combine it with the French and arctic work. Every supposition made in regard to the meridian, supposing that it could be represented by an elliptical curve, seemed to do violence to the results of observation.

A combination was first made, using the arctic and equatorial arcs, the law being that of the square of the sines. This led to a ratio of 214 to 215 for the axes. Then when the middle arc was re-measured, and to the three meridian arcs a longitudi- 
nal one was added this ratio was changed, and 222 to 223 given. The formula was still that of the sines squared. Later, an error was discovered in this remeasurement. Picard had used a toise for his base measures, which was too short by its onethousandth part. The introduction of this new value modified the result so essentially that the law previously adopted no longer satisfied the observations within admissible errors. The formula was changed to one where the increments of the length of the degree varied as the fourth power of the sines of the latitude, and a ratio of 178 to 179 was given for the length of the axes.

The introduction of a power of the sines higher than the square was done reluctantly. But it was found, that in order to repre. sent the curve by the second power of the function, supposing the three arcs subject to the same error, it was necessary to increase the degree in France by sixty-nine toises, and diminish the other two by an equal amount. This would have re-established the ratio 214 to 215 and would have been nearer the truth, as we now know. But such large errors were not thought possible. In fact, reasoning from their accidental errors of observation, only an error of seventeen toises could be admitted for the middle degree, and forty-four for the equatorial one. This would necessitate subtracting one hundred and forty toises from the northern one, which seemed beyond all reason. The procedure, however, wonld make the meridian a perfect ellipse and give a ratio of 250 to 251 .

When compared with Newton's theoretical value of the ellipticity, it was remarked that this erred in defect about as much as the previous conclusion had erred in excess. Therefore the observations left the choice of only two suppositions: either that of the fourth power of the sines, or that of some function of the latitude itself. The arc of longitude which had been measured was brought to bear on the decision, and it was found that the measure would, by the first solution, be in error by one hundred and fifty toises, whereas by the second the error would be reduced to eighteen. This decided the question and the law of the fourth powers, and the ratio 178 to 179 was adopted.

This is the result as given by Bouguer in his discussion of the Peruvian work. Of course it is far from being the truth; but the recapitulation shows to what extent the measures of one hundred and tifty years ago were defective, and gives an idea of the influence of this equatorial arc on the elements of the ellipsoid that are used in all geodetic computations of the present day. A later discussion improved this result, and now there are so many middle arcs entering with great weight on account of their increased accuracy, that the Peruvian arc has 
not the importance it once had. Notwithstanding, it is believed that a remeasurement would so modify it as to materially change the earth's ellipticity.

We now turn to examine the work more in detail. The first base was measured on the plain of Yarouqui, about fifteen miles east of Quito. Eight days were devoted to clearing the line. Its true direction was N. $19^{\circ} 26^{\prime} \mathrm{W}$. Three wooden rods, each twenty feet long, with copper contact plates, pro. jecting one and one-half inches at each end were used in making the measurement. The plates were so arranged as to make contact at right angles to each other. A rope was stretched for alignment, and the inclination of each rod was determmed by means of a level. Twenty-five days were consumed in the work, which was exceedingly laborious because the rods were laid on the ground. This course was pursued on account of the violent wind. Two rods were always in position. The rear one, carried forward by Indians, was brought into contact with as little shock as possible; but with a. heavy rod and in the hands of several untrained persons, it is difficult to see how shocks were avoided. An iron toise was carried along, kept in the shade, and comparisons were made always daily and sometimes oftener. The temperature and humidity of the air affected the wooden rods considerably. The work was begun from both ends and the parties compared their rods when they met in the middle. The south party, however, used tressels, and it was noticed that the effect of wind on the plumb-line, and the consequent error in the length of the base, would be in opposite directions for the two measures. In spite of this fact, and with the exceedingly rough method of making the contact, the two independent measures only differed by about three inches for a distance of more than six thousand toises, which is about $\bar{T}_{\overline{5}} \overline{0}_{\overline{0}}^{\mathbf{1}} \overline{0}$ th part. This is a degree of accuracy far beyond what we can reasonably expect in work of this kind, and there must certainly have been large compensating errors.

Base measures of the present time, with all our improved methods of dealing with the temperature, perfected contact slides, better ways of alignment, and more skillful manipulation of the bars by persons trained to the work, do not give much better results.

The actual measures gave somewhat less than 6273 toises for the length. It was estimated that the necessary corrections would increase this quantity, and in order to have their. base an exact number of toises, one of the end marks was moved three inches and eight lines. It is hard to see what was to be gained by this. Of course the round number would be broken by the solution of the first triangle. Their own measures showed an 
uncertainty of several inches. Therefore, asserting that the base contained an exact number of toises within the thickness of a line, goes for nothing. Moreover the subsequent reduction gave a correction, different from what they had applied, so that the finally adopted lengtl of the base was not 6274 toises, as they wished it to be, but four inches and one and onehalf lines more than this.

The length of the straight line connecting the two extremities of the base was found by tirst comparing the actual measure with the line as traced on the ground, and then deducing the quantity sought from this last line. $\wedge$ approximate value for the base line substituted in the formula*

$$
\mathrm{Z}=\int \frac{c d x}{\left.\sqrt{c^{2}+(b}+\bar{x}\right)^{2}}
$$

gives the correction to reduce the actual measures to the ground line, considering it sensibly straight for each of the seven parts into which the whole base was divided. The absolute and relative heights of the extremities and the intermediate points were determined, which furnished the data for referring the ground line to the air line connecting the extremities. The result of the entire work was:

$\begin{array}{lll}\text { Ground line longer than actual measure ...... } & 1 \cdot 52101 \\ \text { Ground line longer than air line ........... } & 0 \cdot 23100 \\ \text { Air line longer than actual measure ......... } & 1 \cdot 29301\end{array}$

The correction for temperature applied to the Tarqui base would indicate a coefficient of expansion of 000015 for the wooden rods, which is between that of glass and brass but somewhat nearer the latter. But then not very much reliance can be put on the temperatures. That of the base of verification was only estimated, $\uparrow$ and could not certainly liave been known within several degrees. But the accordance of the results, errors of compensation being disregarded, would indicate that the temperatures were correct to within one-fourth of a degree. An examination of the record shows conclusively that this could not liave been the case. On the other hand the Spanish officers correct the second base by about eight feet which would require a coefficient of expansion, based on the same difference of temperature, at least twice that given above. $\neq$

* The earth's radius is $c: x$ is the length of the line, and $b$ an auxiliary constant.

+ Mesure des trois premiers degrés du Méridien, par M. de la Condamine, Paris, 1751 , p. 83 ; see also in this connection "Zeitschrift für Iustrumentenkunde," August, 1885, p. 271. and "Resultate ,über die peruanische Gradmessung." Monat. Corresp., 1887, p. 240.

$\ddagger$ Observaciones astronómicas y physicas hechas de órden de S. M. en los regnos del Perú. Por Juan y Ulloa. Madrid, 1748, p. 166. 
It then appears that the temperature factor alone would give rise to uncertainties far greater than the difference between the two results.

The angles of the triangulation were measured with quad. rants whose radii varied from two to three feet. Two telescopes were provided, a fixed and movable one, the whole instrument being universally mounted by means of two rightangled cylindrical elbows. Micrometers were here applied to instruments of this kind for the first time, and it is believed that Bouguer was the first who called attention to errors of eccentricity. As the limb of the instrument only included ninety degrees these errors could not be studied by the method now employed of comparing diametric readings throughout the entire circle. Independent measures of two known angles gave two equations, in which the known quantities were the errors, and a function of the angle itself, and the unknown quantities were the rectangular coördinates of the center of rotation, referred to the center of graduation as the origin. These coördinates being known from the solution of the equations, corrections applicable to any part of the limb could be calculated. Besides this, six or seven angles, which together closed the horizon, were measured. These were corrected for inclination and their sum compared with $360^{\circ}$. The error of closing was on the average about two minutes. Measures of equilateral triangles gave an additive correction of $20^{\prime \prime}$ for an angle of $60^{\circ}$. Other combinations showed a correction of $40^{\prime \prime}$ for $90^{\circ}$. The separate spaces of five degrees were examined by comparing with a known angle of this magnitude. A month was devoted to the study of the errors of the instrument.

With instruments capable of this degree of accuracy we cannot expect a close agreement between the measured and calculated base. They differ by about two feet. The triangulation is two hundred miles long and contains thirty-two principal triangles. But the result of the side computations from this principal network was twice inodified. Once at the eighth triangle where some auxiliary figures gave a result different by two and one-half toises from the regular work, and again at the sixteenth, where results having a range of seven-tenths of a toise were obtained. from three different methods of deriving the same line. In both these cases the auxiliary work was combined with the regular triangulation, and the resulting line upon which the succeeding work depended, was changed by one and one-tenth toises in the first instance, and by threetenths of a toise in the second; so that we should not be surprised at a much greater discrepancy between calculation and observation at the end of the chain of triangles. Bouguer shows that admitting an error of $15^{\prime \prime}$ in each angle the accu- 
mulation of these throughout the entire work would produce an error in the second base of about twenty-five toises but adds that a certain compensation must be expected among so many errors.

After having finished the triangulation, astronomical observations were undertaken to determine the amplitude of the arc. They were made with a sector having a radius of twelve feet and a graduated arc of about three degrees. Although this is confessedly the weakest part of all the equatorial work, the methods employed show a keen appreciation of many sources of error. The limb was graduated by laying off an aliquot part of the radius as a chord. This was chosen with reference to the particular star to be observed, and the true zenith distance was found by applying to this known arc, a small micrometer correction. The modern work with the zenith telescope is but a repetition of this same principle; for here the absolute zenith distance of the two stars is for the moment disregarded, but the excess of one over the other is measured, and applied to a function of their declinations, which are quantities determined by other investigations.

The precaution was taken in the Peruvian work to make part of the measures on the same star, and at the same time, at both extremities of the arc. This would eliminate any effect of uncertainty in the constants for precession, aberration and nutation, which were not, at that time, very well determined. But Zach has re-reduced the observations of 1742 and 1743 , * and finds a difference of less than $1^{\prime \prime}$ between the results for the simultaneous observations and that deduced from all the work during these two years. The instrument was reversed several times, thus giving values under different conditions, and it is said that no discordant observations were rejected. $\dagger$ The method of reversal is referred to as having been invented by Picard, and it is probable that this principle, now so often applied, and so essential in all instrumental work was here sys. tematically used for the first time. Its effect in this case was to eliminate the eccentricity of the zero point of the micrometer.

The value of the micrometer was found from terrestrial measures, using the known length of portions of the base, and lines erected perpendicular thereto. The meridian was found by observing, at the moment of culmination, the direction of a beam of sunlight, admitted through a hole in the roof of the observatory. The method was supposed to give the true direction with an error not larger than one minute of arc.

* Ueber dic Cradmessung am Aequator; Monat. Corres. vol. xxvi, page 39.

† Figure de la Terre par Bouguer, Paris, 1749, p. 262. 
The accuracy of the measurement of a star's zenith distance appears to depend principally on the stability of the limb of the instrument, and the ability of the observer to set the initial point of the arc in exact coincidence with the plumb-line. There seems to be nothing said as to how this was accomplished, but it is easily seen that it must have been a work requiring much care. The measures of the star's zenith distance are given to the nearest second. Indeed the three results for the arc's amplitude have a range of only three seconds. The following were the results from the three stars:

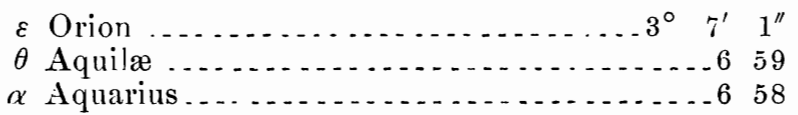

These are the results considered by the observers to be the best. They do not represent all the observations, but were selected on account of the favorable conditions obtaining at the time they were made. The sector had also undergone some improvement. But a mean value from all the results obtained at both stations gives $58^{\prime \prime}$ which agrees more closely than one would expect from the range of the individual values. Zach estimates that the total error in the equatorial degree will not exceed fifty toises, thirty-eight being for the astronomical and twelve for the geodetic part of the work (Monat. Corres. 1807). This is based, however, on Bouguer's, and La Condamine's estimates of the accuracy of the astronomical work (page 251). Since the radius of the sector was twelve feet, one second on the limb would be about 1/1400th of an inch. To make a plumb-line, suspended freely, coincide witl a mark on a scale at its side, to within less than this quantity, must have been a matter of difficulty. In this operation we have in all probability the source of the largest discrepancies.

The flexure of the sector was also studied. Experiments were made on an iron bar, from which it was concluded that the flexure varied as the fourth power of the length. It was found moreover that when the radius of the sector was placed horizontally its flexure amounted to one-twelfth of an inch. This was shown to be inappreciable when the inclination was only a few degrees, and when the objective of the telescope was attached to the center of the sector.

Azimuth observations were made at both bases and at three intermediate points. The agreement between the observed directions and those determined by triangulation is always within less than one minute; the discrepancy at the last base being forty seconds. The sun was invariably used, and the angle between it and a signal was measured with a quadrant. The error's in orientation, estimated liberally, will not change 
the total length of the arc more than a fraction of a second at each end. Therefore the question of azimuth is not one of vital importance.

We now come to perhaps the most interesting part of Bouguer's work. Not satisfied with investigating the exterior sliape of the earth, he determined to study also its interjor condition. It had been known for more than fifty years, that a pendulum oscillated more slowly at the equatur than near the pole, and finding himself not only in a latitude where the force of gravity was the least, but also in a country where there were exceptional facilities for the study of this force at great elevations, he deemed it his duty to devote some time to the investigation of the subject. Of the two methods, either comparing the times of oscillation by the same pendulum, or comparing the lengths of two different pendulums, vibrating in the same time, he chose the former. In this he has been followed by all later observers. Of course his results cannot now be regarded as of very great value, both on account of the unsuitable methods and inferior instruments employed. But the work pointed in a certain direction, which has been confirmed in a general way by some more recent and accurate determinations. His method of getting time would not now be used in gravity observations, although modern instruments would increase the accuracy of the result several fold. It is doubtful whether, with his instruments, and often using single altitudes of stars, the time was correct within several seconds. About one and one-half hours may be taken as the duration of a swing, so that with the uncertain clock correction, and the short duration of the experiment, great discrepancies in the individual swings were unavoidable The pendulum was of an inaccurate type and its length was found by simply holding an iron bar by the side of a thread stretched by an ounce weight. Contact with the clamp above and the bob below was examined either by means of a magnifying glass or by the naked eye.

The individual results, for the length of the seconds pendulum at Quito, where the conditions were favorable, have a range of about $1 / 6000$ part. This wonld correspond to discrepancies in the times of one oscillation of less than one unit in the fourth place of decimals. Under the circumstances the accordance is good.

The result was corrected for buoyancy and temperature. The former was here applied for the first time in pendulum observations. It was estimated that the density of the air on the top of Pichincha, was one eleventh thousandth of that of the metal composing the pendulum bob, and since gravity varies inversely as the length of the seconds pendulum, the length found was 'increased in this same ratio. No correction seems to have been made for the amplitude of oscillation. 
When the necessary reductions were made it was found that gravity at the sea level, was diminislied by 1/1331 part at Quito, and by $1 / 8 \pm 5$ part on the summit of Pichincha. Since the distance from the earth's center had been increased in the first instance by its $1 / 223{ }^{\prime} \prime$ part and in the second by its $1 / 1348$ part the results indicated a law, not very different from that of the inverse square of the distance. But gravity had not changed enough, in either case, to satisfy the law. The conclusion therefore was, that some influence, not exactly understood, increased the force of gravity in both cases. Naturally, attention was drawn to the high table land lying between the stations and the sea. It was estimated that the effect of this would be one-lialf of that of a shell of matter of the same density and thickness encircling the whole earth. Granting this, the diminution of gravity in passing from the sea to the summit would be

$$
\frac{2 h}{r}\left(1-\frac{3 \delta}{4 \Delta}\right)
$$

where $h$ is the height of the station above the sea, $r$ is the radius of the earth and $\delta$ and $\Delta$ are the respective mean densities of the table land and earth.

Now this diminution was found by the pendulum to be $1 / 1331$, which, compared with the above expression, leads to the conclusion that the matter composing the table-land has only about one-fifth the density of the eartl. 'T'he result was something of a surprise at the time, and doubts began to arise as to whether the interior of the earth could be, as some supposed, a fluid mass surrounded by a thin shell. It could not be denied that the density of the surface was less than that of the interior, because it was shown that, in order that their densities be at least equal, the length of the second's pendulum must be in error by about one-thirtieth of an inch, which even with the rough method employed was too great an error to be admitted.

If the land lying between the upper station and the sea be regarded as a plain of infinite extent the same result ensues, and the formula deduced from this point of view is of somewhat simpler derivation. Clarke arrives at the same result by regarding the intervening matter as either a cone, cylinder, or segment of a spliere, where the horizontal dimensions are great compared with the vertical ones. In calculating some attractions in the Hawaiian Islands, the matter was treated rigorously as a cone, and the resultant attraction at the foot of the mountain, based on this value, agreed closely with that derived independently by the latitude observations and triangulation. 
The value of the radius of the earth employed in the Pernvian investigation was about 12,000 meter's too large. Introducing the value now accepted we get a density for the Andes somewhat greater. The change is in the right direction but it is not enough. The rocks in Peru probably have a density of about 2, or possibly less, and if the sea level is in error by one hundred toises, the pendulum work would give about this density for the underlying mass.

The method used in finding the absolute height of the base line, to which all the elevations were referred, was by triangulation. The results were roughly checked by the barometer. From Niguas, a point between Quito and the mouth of the Inca river, angles of elevation were taken to several mountain peaks, of which Piclincha was one. Niguas was also visible from a point near the sea level. The distances being approximately calculated, with some of the angles concluded, the elevations conld be determined with some degree of accuracy. The last station was estimated from barometer readings to be about thirty toises above the sea. But that not much confidence could be placed in the instrument is plain from the fact, afterward stated, that weighing all circumstances it was concluded to fix the difference at forty or forty-two toises. The result was checked by a very rough estimate of the inclination of the river bed and the velocity of the current. Knowing the relation between the velocity and inclination at a point near the station, and determining the velocity farther down the stream, the inclination was calculated. Then from the measured horizontal distance and the inclination the vertical height resulted. It is evident that not much reliance is to be put in such a determination, but perhaps the error is considerably inside some others entering into the deduced height of Pichincha.

The angles of elevation were measured with a quadrant which might give results as much as $30^{\prime \prime}$ from the truth. Then, as there were mountains back of the station, twelve thousind feet high in one case, and fifteen thousand in the other, the angles of elevation may have been in error in either case by the greater part of a minute. And errors from attraction would be accumulative, since Niguas is on the mountain flank. The distance from the sea to Ilinissa, with which Pichincha was connected, was found from the known difference of latitude and the azimuth. It seems therefore probable that the total elevation may have been in error by as much as fifty toises. This is not enough to bring the mean density of the Andes into tolerable accord with that of the surface rock.

It is difficult to accurately estimate the probable error of the distance between the two extremities of the arc, because 
sufficient data are not available. Take one-eighth of a foot, which is one-half the difference between the results, as the probable error of one measure of the base line. This is composed of errors in the lengths of the rods and errors of measures properly so called. The error in the entire base, as depending on the former, varies as the length, and as depending on the latter, as the square root of the length. Assume these to be equal. This would give for the uncertainty of one of the rods (twenty feet) $0 \cdot 0004$ inches or less than $1 / 500000$ th part, and for the uncertainty of making contact about $1 / 500$ of an inch.

Either of these errors is not only much smaller than we can expect from work done under the circumstances, but they are actually less than are gcnerally realized in modern measures. Therefore when we consider the means of comparison with the standard and the method of placing the bars on the ground, the close agreement must be considered entirely accidental, and in no wise to be taken as a criterion of the accuracy of the work.

Any error in the linear measure is transmitted through the triangulation and the probable error in the last side will depend on the average correction to a direction as determined from the shape of the triangles and their number. To this is to be added the error in the base, which transmits itself independently, and its effect depends on the relation between the base and the last side. The average direction error, resulting from joining points in a triangulation, is about twice as much as the average direction error arising from closing the horizon at any one point. Regarding the probable error's of the base and angles as differentials of those quantities, the uncertainty of any side may be computed by a formula involving these differentials and known functions of the angles.

Taking eight seconds as the probable error of an angle which is less than that estimated by the observer, we calculate the uncertainty of the last side, as depending on the angle equations alone to be slightly more than ten feet. This result is based on the formula,

$$
r_{\circ}=a r \sin 1^{\prime \prime} \sqrt{\cot ^{2} \mathrm{~A}+\cot ^{2} \mathrm{~B},}
$$

which assumes that one of the angles in each triangle is a concluded one. The true probable error, where all the angles are measured, would be somewhat less than that given. Never. theless, all the circumstances being considered we may assume the uncertainty of the last line to be not far from twelve feet. The chances are that this is an under-estimate. This error, as we have seen, is about that discovered near the middle of the chain, and which influenced all subsequent work by one-half its 
amount. The error in the base is now disregarded because, although it is much larger than the results of the measures would indicate, its effect on the last side would still be small in comparison with that resulting from the angle equations.

The astronomical olservations agree among themselves, but it was not suspected at the tine, that the mountains might affect the plumb-line by at least thirty times as much as the results were supposed to be in error. When the work was done instruments and methods had not been brought to that degree of perfection necessary to detect these small influences. Since then many striking cases have been brought to light, $22^{\prime \prime}$ deviation having been noticed in India, $16^{\prime \prime}$ in Russia, and $29^{\prime \prime}$ in the Hawaiian Islands. In the example near Moscow there are no mountains to account for the phenomena, and the supposition is that the density of the underlying strata may be subject to great variations, or that large subterranean caverns may exist. Archdeacon Pratt has shown that small changes of density, if extended over a considerable area, may produce very perceptible deflections of the vertical. The Indian example is produced by the Himalayas. The Hawaiian is the result of the attraction of Haleakala, an extinct volcano ten thousand feet high.

When we consider that between the extremities of the Peruvian arc there is a continuous range of mountains, varying in height from nine thousand feet on the plateau of Cochesqui, to nineteen thousand at the summit of Chimborazo, and remember that the arc was terminated at a point where the elevation dropped suddenly several thousand feet, it is evident there must have been enormous differences between the astronomical and geodetic latitudes.

Judging from analogy with other cases, similar, either in the volume of the mountains or the density of the matter, it seems not unlikely that the amplitude of the arc may be in error by many seconds. Indeed if we take the data used in La Place's first discussion, the Peruvian latitudes should be changed by about $10^{\prime \prime}$ in order to give an ellipticity conforming reasonably with our present value. And the required change shows that the plumb-line was drawn toward the mountains.

The error's in the measures of the two bases, in the triangulation, in the altitudes, or in the azimuths, could not have an influence at all comparable to this, so that a simple redetermination of the latitudes would very much improve the result. In fixing the figure of the earth an equatorial arc enters with great weight, and we find that in a combination by least squares of nine arcs used by La Place, an error of one minute in the amplitude of the equatorial arc would reduce the ellipticity to one-half its original value. This seems to be a great 
change for the supposed error, but it must be remembered that not only is the arc at the equator and therefore has great influ. ence in the determination of the elliptic figure, but also that it is a comparatively short arc, and hence any error in the amplitude has a proportionately greater effect on the length of a degree deduced therefrom.

The individual influence of arcs where many enter into the determination should not, however, be overestimated. If we suppose arcs of one degree to be measured from the pole to the equator, say $10^{\circ}$ apart, their weights in fixing the polar axis are approximately as the number's $39,43,54,70,89,111$, $131,146,157,161$ and in the determination of the equatorial axis these same numbers apply in an inverse order. A curve plotted on rectangular coördinates, with the earth's radii and the above weights as arguments, has a point of inflection in middle latitudes, and since the ellipticity is unity minus the ratio of the two axes, middle arcs have very little influence on the ellipticity.

The pendulum observations indicate that the density of the mountains is about one-fifth the mean density of the earth. We may therefore assume that the Andes in the neighborhood of Quito are one-half as dense as the general surface of the earth ; and if we take $15^{\prime \prime}$ for the deflection at each end of the arc the ellipticity of the figure is changed by about one-fourth part of itself.

The effect of any change in an equatorial arc, on the figure of the earth, as deduced from the nine arcs above mentioned is easily found. The conditional equations are combined by least squares in order to find the values of $\mathrm{M}$ and $\mathrm{N}$ in the equation,

$$
d=\mathrm{M}+\mathrm{N} \sin ^{2} l
$$

where $d$ is the length of one degree in latitude $l, M$ is the length of one degree at the equator, and $\mathrm{N}$ is the difference in length between the equatorial and polar degree. The change in length of the equatorial degree will be given by differentiating an expression of the form

$$
\frac{\sum b^{2}\left(\sum a-\sum a b\right)}{9 \sum b^{2}-\left(\sum b\right)^{2}}
$$

where $a$ is the independent variable. The degree being at the equator, the differential of $\Sigma a b$ is zero, and the change in the length of the equatorial degree from the solution of the normal equations would be about two-thirds the assumed linear error in the individual arc. Knowing the differentials of $\mathrm{M}$ and $\mathrm{N}$ the changes produced in the eccentricity and ellipticity are obtained without difficulty. 
It is a singular fact that the first combination of the Lapland and Peruvian ares gave a value for the ellipticity quite as near the truth as was deduced by La Place fifty years later, using the accumulated data furnished by improved instruments and methods. This is partly owing to the fact that the two arcs, having the greatest influence from their position and length, remained mnaltered.

La Place's combination gives a value in excess, and the supposition of the plumb-line being deflected toward the center of the arc changes the value in the right direction. How much attraction should be allowed for it it is difficult to say as the configuration of the land is not known with sufficient accuracy. The indications are, that even admitting the small density of the mountains, the deflections are much larger than would be necessary to bring this arc into accold with the others, and give a value for the ellipticity called for by modern observations in middle latitudes. If we accept the data in La Place's fir'st combination a deflection of eleven seconds at each end of the arc would be required for this purpose.

There seems to have beeu some compensation of errors, which has given the Peruvian arc a value conforming closely with our present spheroid. But its agreement in this respect is no excuse for not remeasuring it. The measures of the base line agree within a few inches, but no one who has examined the case believes that this is anything but an accident. The combination of this arc with some other recent ones in the determination of the figure of the earth, gives corrections for the equatorial latitudes even smaller than those required by points whose positions were determined with greater precision, and where the direction of the plumb-line is much less disturbed by attraction. These small corrections would probably not be confirmed by a new measure.

Notwitlıstanding, this work was well done considering the circumstances and the state of science at the time. Bouguer and his associates were scientific men who thoroughly understood the requirements of the case, and executed the work with the utmost fidelity. 'The necessity for a greater' or less degree of accuracy, according to the kind of observations, and the bearing of each partial result on the final one was the source of constant study. Many principles of work here practiced for the first time have been adhered to by all later observers. But the advantage of repeating this work would come from the great improvements in instruments, and the consequent bringing to light of influences that were then unknown. Nothing at the time was known of spherical excess in geodesy. The theory of least squares was undiscovered, and the method of equal zenith distances had never been applied to the determin- 
ations of latitude. We now have also the compensating base apparatus and many perfected forms of the pendulum for the measurement of the force of gravity. In fact in every class of work the error's at present range from one-tenth to one-hundredth of what was then considered admissible.

Add to this that there is no check on the astronomical latitudes, which are doubly important on account of the shortness of the arc; that the elevation above sea is very uncertain; that their own observations show an uncertainty of seven or eight feet in the sides of the triangles, and that the arc enters into the determination of the ellipse with great effect owing to its geographical position, and it must be conceded that the geodetic science of to-dav demands the re-measurement of the Peruvian arc. It is high time that the equatorial work be put on the same footing as the other data entering into this important problem.

U. S. Coast and Geodetic Survey, Washington, D. C. 